\title{
Análisis filogenético de secuencias de Cistein-Proteasas de Tritrichomonas foetus: ¿salto de la barrera de especies?
}

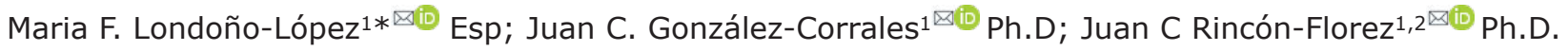

1Universidad Tecnológica de Pereira, Facultad Ciencias de la Salud, Programa Medicina Veterinaria y Zootecnia, Grupo de investigación Grupo BIOPEC: Biomolecular y Pecuaria, Pereira - Risaralda, Colombia.

2Universidad Nacional de Colombia, Sede Palmira, Facultad de Ciencias Agropecuarias, Departamento de Ciencia Animal. Palmira, Valle del Cauca, Colombia.

*Correspondencia: m.Iondono@utp.edu.co

Recibido: Enero 2020; Aceptado: Agosto 2020; Publicado: Noviembre 2020.

\section{RESUMEN}

Objetivos. Realizar un análisis de las secuencias de los genes de las cisteína-proteasas (CP) de Tritrichomonas spp. reportadas en GenBank con el fin de desarrollar un análisis filogenético que ayude a esclarecer la posibilidad de transmisión entre especies. Materiales y métodos. Se realizó el análisis de las secuencias de CP de Tritrichomonas spp. disponibles en GenBank. Las secuencias halladas fueron alineadas, identificando sitios polimórficos. Se determinó el mejor modelo de sustitución nucleotídica y se construyeron árboles filogenéticos por cada gen, usando como grupo externo, Trichomonas vaginalis. Además, se construyeron secuencias en tándem de cada patógeno reportado, para construir árboles filogenéticos de mayor fortaleza en las ramas. Finalmente, se estimó la divergencia evolutiva de las secuencias en tándem. Resultados. El análisis filogenético evidenció la relación que puede existir entre $T$. suis porcino y $T$. foetus bovino. Las secuencias de $T$. foetus felino y bovino se encontraron en grupos separados; sin embargo, las de $T$. foetus felino fueron similares a las de bovinos de Namibia. También se pudo evidenciar la cercanía de $T$. foetus humano con $T$. foetus bovino y porcino. Conclusiones. El análisis filogenético de las secuencias de CP en diferentes especies de Tritrichomonas spp. permitió identificar relaciones entre las Tritrichomonas de bovinos, porcinos y humanos, pero también entre algunas secuencias aisladas de felinos con las de bovinos de Namibia, lo que sugiere la posibilidad del paso de la barrera entre especies.

Palabras Clave: Ganado; protozoarios; parásitos; tritrichomoniasis (Fuente:MeSH).

\section{ABSTRACT}

Objectives. To analyze the cysteine-protease (CP) gene sequences of Tritrichomonas spp. reported in GenBank, and develop a phylogenetic analysis to help clarify the possibility of transmission between species. Materials and Methods. The CP sequences of Tritrichomonas spp. available in GenBank were analyzed and aligned to identify polymorphic sites. The best nucleotide substitution model was determined, and phylogenetic trees were constructed for each gene, using Trichomonas vaginalis 
as an outgroup. Besides, tandem sequences of each reported pathogen were constructed to build phylogenetic trees with higher branch strength. Finally, the evolutionary divergence of the tandem repeat sequences was estimated to obtain more conclusive results. Results. The phylogenetic analysis showed the relationship that may exist between porcine $T$. suis and bovine $T$. foetus. The feline and bovine $T$. foetus sequences were found in separate groups; however, feline $T$. foetus was similar to that of bovines from Namibia. The proximity of human $T$. foetus to bovine and porcine $T$. foetus was verified. Conclusions. Phylogenetic analysis of CP sequences in different species of Tritrichomonas spp., identified relationships between bovine, porcine and human Tritrichomonas, but also between some isolated feline sequences with those of bovines, suggesting a possibility of barrier crossing between species.

Keywords: Cattle; protozoan; parasite; tritrichomoniasis (Source: MeSH).

\section{INTRODUCCIÓN}

La tritrichomoniasis es una infección ocasionada por el parásito protozoario Tritrichomonas spp., que pertenece al orden Trichomonida de la familia Trichomonidade de la clase Parabasalia (1). Las tritrichomoniasis son infecciones comunes en órganos reproductivos en bovinos, siendo su principal transmisión la vía genital, dando como resultado muerte embrionaria, infertilidad y abortos (2). En los porcinos este parásito puede invadir el estómago, ciego y cavidad nasal (3).

Recientemente, se ha determinado que puede ocasionar infecciones el tracto intestinal en gatos, específicamente en el lumen y cripta del epitelio de la mucosa colónica, induciendo efectos citopáticos en las células epiteliales, dando como resultado, diarreas crónicas de difícil tratamiento (4). La adherencia, colonización y patogenicidad de la $T$. foetus, se da por la Cisteín-Proteasa (CP), que es reconocida como mediadora de la citotoxicidad y es la encargada de la adhesión y colonización del protozoario al tejido (5). Estos protozoos tienen expresión de gran número de genes asociados a la virulencia o a su propio metabolismo, pero algunos son altamente conservados. Por ejemplo, las regiones espaciadoras internas transcriptas (ITS ) 1 y 2 son altamente conservadas, lo que las hace poco útiles para realizar análisis filogenético y entender la variación entre especies que puedan estar asociados al hospedero. Por esta razón, es de gran importancia utilizar genes más variables, como es el caso de las CP. Recientemente se ha planteado que la CP2 es las más variables y adecuada para realizar análisis filogenéticos (1).

La Tritrichomonas spp. aislada de porcinos ha presentado controversia en cuanto a su denominación. Anteriormente fue clasificada como $T$. suis, sin embargo, estudios genéticos y filogenéticos demostraron que el protozoo $T$. suis es el mismo $T$. foetus bovino, permitiendo así, la reclasificación a $T$. foetus genotipo porcino (6)1928. Existen reportes de infecciones oportunistas por parte de la Tritrichomonas foetus, en humanos inmunodeprimidos o inmunosuprimidos permitiendo la colonización de la $T$. foetus en sistema respiratorio (7). Sin embargo, todavía no está clara la ruta de infección y el genotipo de estos parásitos de acuerdo a su hospedero (8).

Por lo anterior, el análisis filogenético podría proveer información importante sobre las variaciones del parásito y su relación con el hospedador, el objetivo del presente estudio fué realizar un análisis de las secuencias de los genes de las Cistein-Proteasas (CP) de Tritrichomonas spp reportadas en el GenBank, con el fin de desarrollar un análisis filogenético que aporte al esclarecimiento de la posibilidad de transmisión entre especies.

\section{MATERIALES Y MÉTODOS}

\section{Secuencias cisteín-proteasas} Tritrichomonas spp. Se realizó una búsqueda en el GenBank usando las palabras CisteínProteasa y Tritrichomonas spp, la cual permitió obtener secuencias reportadas para diferentes tipos de Cisteín-Proteasa (CP1, CP2, CP4). Posteriormente se identificó la información de la región geográfica y la especie de la que fue aislada. Para el análisis, Se tuvieron en cuenta solamente los tipos de Cisteín-Proteasa de las que había secuencias de Tritrichomonas spp y que además también hubiera en Trichomonas vaginalis, ya que esta especie actuó como grupo externo en el análisis. Al finalizar la búsqueda, se contó con 70 secuencias para los análisis posteriores, de las cuales 18 secuencias fueron de CP1, 33 de CP2 y 19 de CP4. Adicionalmente, 
se incluyó una secuencia de CP1, CP2 y CP4 de Trichomonas vaginalis (outgroup).

Edición y análisis filogenético de secuencias. Las secuencias fueron editadas usando el programa Molecular Evolutionary Genetics Analysis v.10.1.8 (MEGA v.10.1.8.) y se realizó un alineamiento para cada una de las proteasas CP1, CP2 y CP4 reportadas en el NCBI, usando el algoritmo MUSCLE del programa MEGA v.10.1.8. A partir del alineamiento se realizó la edición y un análisis descriptivo de las secuencias donde se tomaron los estadísticos relacionados con las regiones variables y conservadas y se determinó el mejor modelo de sustitución nucleotídica usando el menor valor, según el criterio de información bayesiano (BIC), estimado mediante el programa MEGA v.10.1.8.

El análisis filogenético se realizó con Bootstrap mediante máxima verosimilitud usando las secuencias nucleotídicas de cada una las CisteinProteasas (CP1, CP2, CP4), usando el mejor modelo de sustitución nucleotídica obtenido en cada caso y anotando la información del país de origen y la especie de la que fue aislada, con el fin de buscar posibles patrones de asociación. Se usaron 1000 repeticiones bootstrap y se consideraron significativos los valores superiores al $70 \%$.

Construcción de secuencias en tándem. A partir de la información anterior, se contruyeron secuencias en tándem de las Cistein-Proteasas $\mathrm{CP} 1, \mathrm{CP} 2$ y CP4 de las Tritrichomonas spp reportadas y de la Trichomonas vaginalis como outgroup, teniendo en cuenta también la información del el país de origen y las especies de animales de las que fueron aisladas. Se realizó un análisis filogenético con Bootstrap mediante máxima verosimilitud con el modelo de sustitución $\mathrm{K} 2$ con distribución gama discreta $(\mathrm{K} 2+\mathrm{G})$ y un Bootstrap con 1000 repeticiones. Finalmente, se estimó la divergencia evolutiva de las secuencias en tándem mediante el programa MEGA v.10.1.8.

Aspectos éticos. En el presente artículo no se realizaron procedimientos experimentales con animales; solo se realizó un análisis de información reportada. El Proyecto fue aprobado por el comité de bioética de la Universidad Tecnológica de Pereira - CBE-UTP (Pereira, Colombia; código CBE-SYR-162016).

\section{RESULTADOS}

Las secuencias reportadas de interés fueron tres tipos de Cistein-Proteasas de Tritrichomonas spp., aisladas de diferentes países de cuatro continentes y de diferentes hospedadores como bovinos, porcinos, felinos, primates y humanos (Tabla 1). Las secuencias reportadas para Latino América fueron principalmente de bovinos y no se encontraron secuencias de gatos para América Latina. Los registros y accesos para cada secuencia se presentan en la tabla 1.

Tabla 1. Listado de secuencias de Cistein-Proteasas

de Tritrichomonas spp. reportadas en el GenBank, con número de registro, especie animal del aislado, país de origen y código de acceso en el GenBank.

\begin{tabular}{|c|c|c|c|c|c|}
\hline Gen & $\mathbf{N}$ & Especie & Hospedero & País & Acceso \\
\hline CP1 & 18 & $\begin{array}{l}\text { T.suis } \\
\text { T. foetus } \\
\text { T. mobilensis } \\
\text { Trichomonas } \\
\text { vaginalis }\end{array}$ & $\begin{array}{l}\text { Porcine, } \\
\text { Feline, Bovine, } \\
\text { Saimiri boliviensis, } \\
\text { Homo sapiens } \\
\text { Human }\end{array}$ & $\begin{array}{l}\text { Argentina, Australia, } \\
\text { Bolivia, Czech } \\
\text { Republic Germany, } \\
\text { Japan, Namibia, USA, } \\
\text { Switzerland. } \\
\text { Unated Kingdom }\end{array}$ & $\begin{array}{c}\text { JX648147.1, JX187024.1, JX648146.1, JX648148.1, } \\
\text { JX187017.1, JX187015.1, JX187016.1, JX187014.1, } \\
\text { JX187013.1, JX187018.1, JX187020.1, JX187019.1, } \\
\text { JX187022.1, JX187021.1, KX425904.1, KX425905.1, } \\
\text { LC054290.1, LC054281.1. } \\
\text { X77218.1 }\end{array}$ \\
\hline $\mathrm{CP} 2$ & 33 & $\begin{array}{c}\text { T.suis } \\
\text { T. foetus } \\
\text { T. mobilensis }\end{array}$ & $\begin{array}{c}\text { Porcine, Feline, } \\
\text { Bovine, Saimiri } \\
\text { boliviensis } \\
\text { Homo sapiens }\end{array}$ & $\begin{array}{l}\text { Argentina, Australia, } \\
\text { Bolivia, Czech } \\
\text { Republic, Germany, } \\
\text { Japan, Namibia, USA, } \\
\text { Switzerland }\end{array}$ & $\begin{array}{c}\text { JX187040.1, JX648149.1, JX648150.1, JX648151.1, } \\
\text { KP012652.1, JX187026.1, JX187027.1, JX187028.1, } \\
\text { JX187029.1, JX187030.1, JX187031.1, JX187032.1, } \\
\text { JX187033.1, JX187035.1, JX187036.1, JX187038.1, } \\
\text { JX187039.1, JX187040.1, KX425891.1,KX425892.1, } \\
\text { KX425893.1, KX425894.1, KX425895.1, KX425896.1, } \\
\text { KX425897.1, KX425898.1, KX425899.1, KX425900.1, } \\
\text { KX425901.1, KX425902.1, KX425903.1, LC054282.1, } \\
\text { LC054291.1 } \\
\text { X77219.1 }\end{array}$ \\
\hline CP4 & 19 & $\begin{array}{l}\quad \text { T.suis } \\
\quad T . \text { foetus } \\
\text { T. mobilensis } \\
\text { Trichomonas } \\
\quad \text { vaginalis }\end{array}$ & $\begin{array}{l}\text { Porcine, Feline, } \\
\text { Bovine, Saimiri } \\
\text { boliviensis } \\
\text { Homo sapiens } \\
\text { Human }\end{array}$ & $\begin{array}{l}\text { Argentina, Australia, } \\
\text { Bolivia, Czech } \\
\text { Republic, Germany, } \\
\text { Japan, Namibia, USA, } \\
\text { Switzerland } \\
\text { Unated Kingdom }\end{array}$ & $\begin{array}{c}\text { JX648154.1, JX648153.1, JX187052.1, KP012653.1, } \\
\text { JX187046.1, JX187045.1, JX187044.1, JX187043.1, } \\
\text { JX187041.1, JX187042.1, KX425907.1, KX425906.1, } \\
\text { JX187051.1, JX187050.1, JX187049.1, JX187048.1, } \\
\text { JX187047.1, JX187053.1, LC054283.1 } \\
\text { X77221.1 }\end{array}$ \\
\hline
\end{tabular}


A partir del análisis descriptivo del alineamiento de las secuencias de Tritrichomonas spp, se pudo evidenciar que la secuencia para CP4 fue la que presentó menos sitios variables (2), y las secuencias CP1 y CP2 las que más sitios variables presentaron ( 4 y 28 respectivamente). El alineamiento más largo fue para la secuencia CP2 con 669 nucleótidos y el más corto para la CP4 con 273 nucleótidos.

El porcentaje de GC mayor fue el de la CP4 con un rango entre $20.17 \%$ y $24.55 \%$ y el de menor valor para la CP1 con un rango entre $21.77 \%$ y $14.79 \%$. Las secuencias en tándem presentaron un tamaño de 2283 nucleótidos con 48 sitios variables, 2235 conservados y un porcentaje GC entre 22.60 a 21.24 (Tabla 2). En general el mejor modelo de sustitución nucleotídica fue kimura 2-parámetros (K2), pero en el caso de las secuencias CP2 incluyo el parámetro I $(\mathrm{K} 2+\mathrm{I})$ que asume que cierta fracción de sitios son evolucionariamente invariables. Además, la secuencia en tándem incluyó el parámetros $\mathrm{G}(\mathrm{K} 2+\mathrm{G})$, que usa una distribución Gamma discreta para modelar las tasas evolucionarias no uniformes.

A partir de los reportes del mejor modelo de sustitución, se realizó el análisis filogenético que permitió evidenciar una topología muy similar en los árboles construidos de las diferentes proteasas evaluadas, con una clara separación de la Trichomonas vaginalis del resto de las Tritrichomonas spp. analizadas. Adicionalmente, se pudo identificar una agrupación entre las Tritrichomonas foetus de felinos en clados diferentes a las de bovinos, porcinos y humanos. Llama la atención la agrupación de las $T$. foetus de bovinos de Namibia en el clado de la $T$. foetus de felinos, y muy cerca a ellos la $T$. mobiliensis, siendo el bovino más susceptible a los diferentes tipos (Figura 1).

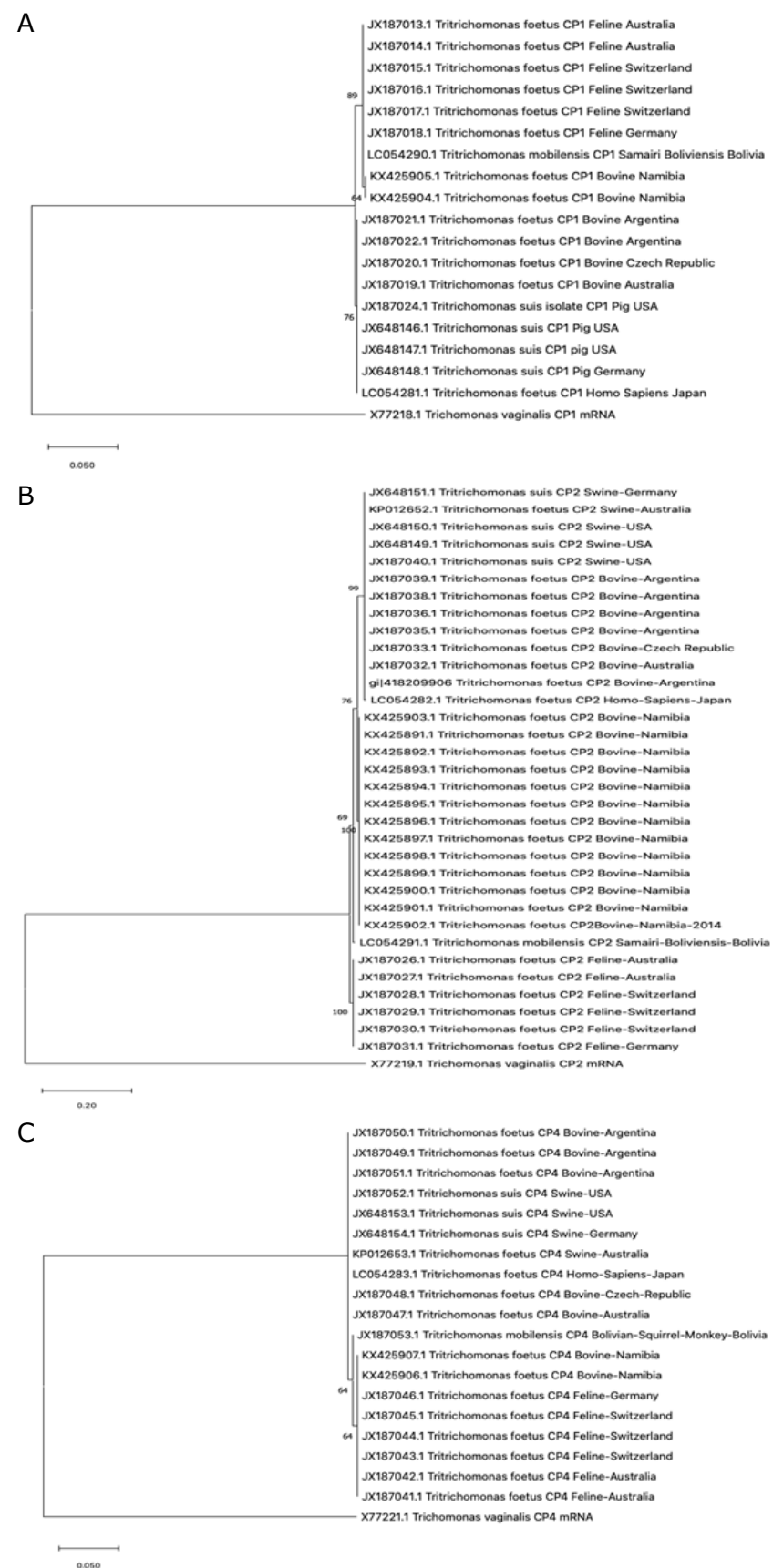

Figura 1. Árboles filogenéticos por máxima verosimilitud de secuencias de CisteinProteasas de Tritrichomonas spp de acuerdo a: A) CP1, B) CP2 y C) CP4, usando Trichomonas vaginalis como outgroup.

Tabla 2. Estadísticos para los alineamientos de secuencias de Cistein-Proteasa de Tritrichomonas spp. y mejor modelo de sustitución nucleotídica.

\begin{tabular}{ccccccc}
\hline Proteasas & N & Sitios Variables & Sitios Conservados & Nucleótidos & Modelo de Sustitución & Porcentaje G-C (\%) \\
\hline CP1 & 18 & 4 & 499 & 503 & K2 & $21.77-14.79$ \\
CP2 & 33 & 28 & 641 & 669 & K2+I & $22.22-21.77$ \\
CP4 & 19 & 2 & 271 & 273 & K2 & $20.17-24.55$ \\
Tándem & 12 & 48 & 2235 & 2283 & K2+G & $22.60-21.24$ \\
\hline
\end{tabular}

G-C: Guanine-Cytosine 
Vale la pena resaltar que los diferentes Tritrichomonas spp., se agrupan entre si dependiendo de los países de los que fueron aislados, pudiendo observar así una clara similitud filogenética en los patógenos de un mismo país, lo que plantea la posibilidad de evidenciar potenciales entradas de la enfermedad y sus probables relaciones (Figura 1 ).

Finalmente, la secuencia en tándem pretendía dar una mirada global de las relaciones a partir de los tres genes de Cistein-Proteasas de Tritrichomonas spp., lo que permitió mejorar la fortaleza de las relaciones y se evidencia nuevamente la separación en un grupo, de las Tritrichomonas spp aislada de bovinos y porcinos, diferente al grupo conformado por las Tritrichomonas spp aisladas de felinos, bovinos de Namibia y primates (Figura 2),

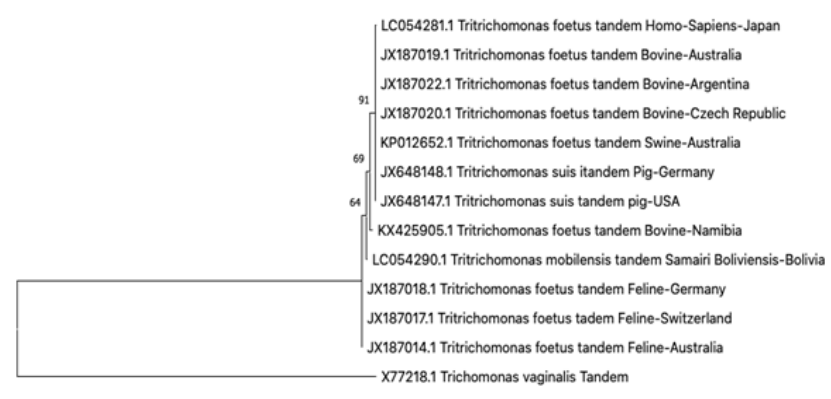

Figura 2. Árbol filogenético por máxima verosimilitud y bootstrap basado en secuencias en tandem de Cistein-Proteasas CP1, CP2 y CP4 de Tritrichomonas spp. y Trichomonas vaginalis como outgroup.

Al realizar el análisis de divergencia evolucionaria de las 13 secuencias construidas en tándem, se pudo soportar los resultados obtenidos con los arboles filogenéticos, donde se puede evidenciar la similitud entre las secuencias aisladas de bovinos, porcinos y humanos, que presentaron muy poca divergencia (0.0), quienes, a su vez son distantes a las de felinos y primates no humanos en donde los valores fueron de 0.023. Las secuencias de Tritrichomonas mobilensis de primates no humanos, que a pesar de ser una especie diferente a la $T$. foetus, se encontró evolutivamente mas cercano a las secuencias de las $T$. foetus de felinos con valores de 0.012 , y mas distante de las de humanos con 0.019. Se destaca que las secuencias de $T$. foetus de felinos resultaron mas cercanas a las secuencias de $T$. foetus de bovinos de Namibia con 0.017 comparandolo con las secuencias de $T$. foetus de bovinos, porcinos y humanos de otros paises con 0.023 .

\section{DISCUSIÓN}

La Tritrichomonas foetus se ha considerado como un parásito común en el ganado bovino en el que causa abortos y en los últimos años se ha identificado en el gato como causante de diarrea crónica (9). En los humanos se encuentran algunos parásitos cercanos exclusivos de la especie, la Trichomonas vaginalis (10) y la Trichomonas tenax (11) como también la Pentatrichomonas hominis (7) que se ha aislado también en animales domésticos; sin embargo se ha detectado a la $T$. foetus como agente etiológico de enfermedad respiratoria y colecistitis. Por otro lado, en el cerdo la Trichomonas foetus, antes llamada Tritrichomonas suis, causa enfermedad respiratoria en el hombre, lo que hace pensar en un mismo agente etiológico para varias especies (12).

Las proteasas son proteínas indispensables para la virulencia del protozoo y la interacción del hospedero-parásito; además para la adquisición de nutrientes, inducción de la apoptosis celular, adherencia, metabolismo e inducción de la inflamación (13). Por este motivo es un buen candidato para la evaluación filogenética. Además, se ha reportado que usando el análisis multigenético de las Cistein-Proteasas de los diferentes genotipos de Tritrichomonas spp. aisladas de felinos, bovinos, porcinos y primates, se encuentran regiones conservadas, que sugieren que los genotipos de las $T$. foetus de bovinos originarios de Namibia y felinos en varios países del mundo se encuentran asociados con la $T$. mobiliensis en primates (6).

Al realizar alineaciones de 70 secuencias de las tres diferentes Cistein-Proteasas de Tritrichomonas spp analizadas y reportadas en el GenBank, se pudo apreciar que las regiones variantes fueron 4,28 y 2 para las CP1, CP2 y CP4 respectivamente, evidenciando una alta variabiliadad de la CP2 que, de acuerdo a Slapeta, esta Cistein-proteasa es la mas variable, siendo la mas adecuada para ser usada en analisis filogenéticos para estos casos. (6). Para obtener una mayor fortaleza en las ramas, se construyó la secuencia tándem con las tres proteasas reportadas para cada especie de Tritrichomonas spp. aisladas de diferentes animales. Las secuencias tándem al ser más largas y presentar todos los sitios variantes 
permitió observar de forma más clara la organización entre los clados del árbol. Además, es importante notar que la Trichomonas vaginalis a pesar de ser clasificados como Trichomonadae, es un protozoo evolutivamente diferente a las Tritrichomonas spp. situación que permitió que se comportarse como un grupo externo en el análisis filogenético y que puede ser observado en la divergencia evolutiva (Tabla 3).

Tabla 3. Divergencia evolucionaria entre las 13 secuencias de Cistein-Proteasas en tándem.

\begin{tabular}{|c|c|c|c|c|c|c|c|c|c|c|c|c|}
\hline Secuencia & 1 & 2 & 3 & 4 & 5 & 6 & 7 & 8 & 9 & 10 & 11 & 12 \\
\hline \multicolumn{13}{|l|}{$\begin{array}{l}\text { 1. LC054290. } 1 \\
\text { T. mobilensis Saimiri bolivensis - Bolivia }\end{array}$} \\
\hline $\begin{array}{l}\text { 2. JX648147.1 } \\
\text { T. suis porcino USA }\end{array}$ & 0.017 & & & & & & & & & & & \\
\hline $\begin{array}{l}\text { 3. JX648148.1 } \\
\text { T. suis porcino Alemania }\end{array}$ & 0.017 & 0.000 & & & & & & & & & & \\
\hline $\begin{array}{l}\text { 4. KP012652.1 } \\
\text { T. foetus porcino Australia }\end{array}$ & 0.019 & 0.002 & 0.002 & & & & & & & & & \\
\hline $\begin{array}{l}\text { 5. LC054281. } 1 \\
\text { T. foetus Homo sapiens Japón }\end{array}$ & 0.017 & 0.000 & 0.000 & 0.002 & & & & & & & & \\
\hline $\begin{array}{l}\text { 6. JX187017.1 } \\
\text { T. foetus felino Suiza }\end{array}$ & 0.012 & 0.023 & 0.023 & 0.023 & 0.023 & & & & & & & \\
\hline $\begin{array}{l}\text { 7. JX187018.1 } \\
\text { T. foetus felino Alemania }\end{array}$ & 0.012 & 0.023 & 0.023 & 0.023 & 0.023 & 0.000 & & & & & & \\
\hline $\begin{array}{l}\text { 8. JX187014.1 } \\
\text { T. foetus felino Australia }\end{array}$ & 0.012 & 0.023 & 0.023 & 0.023 & 0.023 & 0.000 & 0.000 & & & & & \\
\hline $\begin{array}{l}\text { 9. JX187020.1 } \\
\text { T. foetus bovino Republica Checa }\end{array}$ & 0.017 & 0.000 & 0.000 & 0.002 & 0.000 & 0.023 & 0.023 & 0.023 & & & & \\
\hline $\begin{array}{l}\text { 10. KX425905. } 1 \\
\text { T. foetus bovino Namibia }\end{array}$ & 0.013 & 0.015 & 0.015 & 0.017 & 0.015 & 0.017 & 0.017 & 0.017 & 0.015 & & & \\
\hline $\begin{array}{l}\text { 11. JX187019.1 } \\
\text { T. foetus bovino Australia }\end{array}$ & 0.017 & 0.000 & 0.000 & 0.002 & 0.000 & 0.023 & 0.023 & 0.023 & 0.000 & 0.015 & & \\
\hline $\begin{array}{l}\text { 12. JX187022.1 } \\
\text { T. foetus bovino Argentina }\end{array}$ & 0.017 & 0.000 & 0.000 & 0.002 & 0.000 & 0.023 & 0.023 & 0.023 & 0.000 & 0.015 & 0.000 & \\
\hline $\begin{array}{l}\text { 13. X77218. } 1 \\
\text { Trichomonas vaginalis humano, Reino unido }\end{array}$ & 1.036 & 1.057 & 1.057 & 1.059 & 1.057 & 1.020 & 1.020 & 1.020 & 1.057 & 1.040 & 1.057 & 1.057 \\
\hline
\end{tabular}

Al obtener un mejor panorama de la distribución filogenética de los Tritrichomonas $s p p$, y realizando un análisis de divergencia, se encontró similitud entre las secuencias de $T$. foetus de bovinos y porcinos. Este resultado es similar al encontrado por Mueller et al (14) en Australia en donde comparó Cisteín-Proteasa $1,2,4$ y 9 de cerdos y bovinos que convivían juntos y en donde encontró que el genotipo presente en las dos especies era igual. Además, han reportado en los cerdos una prevalencia del $65 \%$ del genotipo bovino a pesar de que esta producción está considerada libre de $T$. foetus en el ganado vacuno por 30 años, lo cual quiere decir que este parásito puede estar presente en los cerdos de las granjas sin producir en ellos enfermedad, ni siendo fuente de contagio a los bovinos (14).
A diferencia de este estudio en donde no se encontró similitud entre el genotipo de $T$. foetus de felinos y porcinos usando las secuencias de Cistein-Proteasas, Doi et al (15) encontraron que los genotipos identificados en pacientes felinos de algunos hospitales veterinarios de Japón eran iguales a los genotipos reportados en el GenBank para porcinos de acuerdo a las regiones TR/7 y TR/8; así mismo encontraron que ambos genotipos fueron distintos a la $T$. foetus del bovino (15), aunque es importante tener en cuenta que se usaron diferentes genes para el análisis, estos pueden ser más conservados y menos relacionados con los mecanismos de invasión, lo que puede explicar conclusiones diferentes, pero aumenta la posibilidad de que se pueda dar el salto de barrera entre especies para diferentes genotipos de Tritrichomonas spp., 
situación que debe ser investigada a profundidad en otros trabajos con mayor número de genes.

En el árbol filogenético, se puede evidenciar que las secuencias de $T$. foetus de bovinos son similares entre ellas y se presenta muy poca distancia genética, con excepción de la secuencia de $T$. foetus aisladas de Bovino en Namibia, la cual presenta mayor cercanía genética con las $T$. foetus felino que se ubica en el mismo clado, y presenta una divergencia genética mínima. Además, llama la atención que las secuencias de $T$. foetus de Bovinos de Namibia y las de felinos se secuencias más cercanas de $T$. mobiliensis, que de las secuencias de $T$. foetus de otros bovinos, de cerdos o de humanos, lo que hace pensar sobre la verdadera clasificación de estos grupos: Es importante resaltar que previamente se había reportado el parecido entre $T$. foetus de los bovinos de Namibia y T. mobiliensis (6) y que $T$ suis ha sido propuesta como un genotipo de $T$. foetus (12), lo que plantea la necesidad de revisar la clasificación de algunos miembros de Tritrichomonas. Lo anterior también plantea que al menos dos genotipos diferentes de $T$. foetus pueden infectar el bovino y que cada genotipo se asocia con especie diferentes, lo que supone una plasticidad del patógeno para pasar entre especies. Sin embargo, aún falta investigación al respecto, usando información de genomas completos aislados de diferentes hospedadores, que permitan aclarar la situación.

Entre las secuencias de $T$. foetus felino se evidencia poca variación entre ellas. Slapeta y colaboradores, coinciden en afirmar que los genotipos de $T$. foetus bovino y felino son diferentes luego de comparar las regiones TR/7 y TR/8 (16); sin embargo Morin et al (17) encontraron una estrecha similitud entre el transcriptoma al comparar ambos tipos de $T$. foetus en un estudio que tenía como objetivo identificar blancos terapéuticos para el tratamiento de este parásito, teniendo en cuenta su adaptación a distintas especies y los cambios en el transcriptoma por esta adaptación. De acuerdo a estos resultados, los autores concluyen que este parásito tiene una reciente adaptación evolutiva al huésped (17). Es importante resaltar que estudios como el de Morin, abren la posibilidad de encontrar blancos terapéuticos específicos dada la dificultad de tratamiento en especies como el felino en el que la $T$. foetus presenta resistencia a la mayoría de antiparasitarios a excepción del ronidazol el cual ha presentado alta toxicidad en los animales tratados (17). Hallazgos similares fueron encontrados por Reinmann et al (18) quienes compararon secuencias ITS 2 y el factor de elongación a 1 como secuencia semi conservada, encontrando que las secuencias de ambos locis son filogenéticamente muy cercanos (18). Estas similitudes abren la puerta a futuros estudios teniendo en cuenta que en muchas producciones bovinas en donde se guarda alimento concentrado, se encuentran gatos para controlar a los roedores que frecuentan los sitios de almacenamiento, con lo que pueden ser estos felinos un foco de contagio para el ganado vacuno.

Por otro lado, en Mantes, Francia en el año 2006; se reportó un caso de neumonía neumocística en una mujer de 54 años producida por $T$. foetus. El diagnóstico del agente etiológico se realizó por medio de PCR de la región ITS 1 y su secuencia comparada con secuencias encontradas en bases de datos. Las secuencias presentaron homología de $99.2 \%$ respecto de las secuencias de $T$. foetus, $T$. suis y $T$. mobilensis encontradas en bovinos, cerdos y ardillas (19). Otro caso reportado en humano fue hecho en Japón, en donde se aisló $T$. foetus de conducto biliar de un paciente con colecistitis. Posterior al aislamiento se realizó genotipificación a las $C P 1,2,4$ y 9 y se comparó con las secuencias de $T$. foetus de ganado y cerdo dando como resultado genotipos iguales (8).

Todos estos resultados alertan sobre la importancia de la $T$. foetus no solo como causante de enfermedad en gatos y animales de abasto sino también de la importancia como posible zoonosis, más si se tiene en cuenta todos los posibles trasmisores hacia el hombre y además porque no se han realizado estudios sistemáticos en humanos que tengan contacto frecuente con las especies susceptibles. A pesar de que algunos estudios muestran que genotipos similares de $T$. foetus se encuentran en diferentes especies sin causar enfermedad (14), otros reportes muestran pacientes como cerdos y humanos con enfermedad respiratoria, problemas intestinales en gatos y reproductivos en bovinos, lo que hace necesario futuras investigaciones en las que se comparen los genotipos de este parásito en las especies mencionadas teniendo en cuenta que es frecuente que producciones tanto bovinas como porcinas tienen gatos en las mismas, además del contacto estrecho del humano con estos animales (20). Por otro lado, al tener solo un reporte conocido en Latino América de $T$. foetus en gatos (1), es fundamental realizar estudios de prevalencia en esta región teniendo en cuenta 
la alta prevalencia en países desarrollados como Alemania, Australia y Suiza (6).

Finalmente, al realizar el realizar el análisis de los árboles por distribución geográfica, se observó una similitud de patógenos de acuerdo a los países donde fue tomada la muestra, lo que supone que estas secuencias pueden ser útiles para llevar a esclarecer el origen de estos parásitos, y la posible contaminación cruzada entre especies animales, por lo que pueden ser consideradas en futuros estudios en Latinoämerica para identificar la posible procedencia del patogeno.

En conclusión, en el presente estudio se pudieron estimar las distancias filogenéticas de las diferentes secuencias de las cisteinproteasas analizadas, sugiriendo cercanía entre las Tritrichomonas foetus aisladas en felinos, bovinos de Namibia y Tritrichomonas mobilensis en primates no humanos, pero un poco más distantes del grupo formado por las $T$. foetus de bovinos, y $T$. suis de porcinos y una secuencia de humanos. Estas cercanías y el hecho de encontrar secuencias de $T$. foetus de bovinos en los dos grupos sugieren la posibilidad del paso de la barrera entre especies. Sin embargo, se requieren estudios moleculares moleculares in vivo con diferentes poblaciones de animales y humanos relacionados entre sí.

\section{Conflicto de intereses}

Los autores declaramos que no tenemos conflicto de intereses.

\section{Agradecimientos}

A la Universidad Tecnológica de Pereira por el apoyo y la financiación del proyecto de investigación con código 5-17-9. Titulado "Factores asociados a la presencia, resistencia a antiparasitarios y fenotipos de Tritichomonas foetus en gatos, humanos y algunos animales de producción del Eje cafetero".

\section{REFERENCIAS}

1. Hora AS, Miyashiro SI, Cassiano FC, Brandão PE, Reche-Junior A, Pena HFJ. Report of the first clinical case of intestinal trichomoniasis caused by Tritrichomonas foetus in a cat with chronic diarrhoea in Brazil. BMC Vet Res. 2017; 13(1):109. https://doi.org/10.1186/ s12917-017-1026-3

2. Mardones FO, Perez AM, Martínez A, Carpenter TE. Risk factors associated with Tritrichomonas foetus infection in beef herds in the Province of Buenos Aires, Argentina. Vet Parasitol. 2008; 153(34):231-237. https://doi.org/10.1016/j. vetpar.2008.01.038

3. Morin-Adeline V, Mueller K, Conesa A, Šlapeta J. Comparative RNA-seq analysis of the Tritrichomonas foetus PIG30/1 isolate from pigs reveals close association with Tritrichomonas foetus BP-4 isolate 'bovine genotype. Vet Parasitol. 2015; 212(34):111-117. https://doi.org/10.1016/j. vetpar.2015.08.012
4. Frey CF, Müller N. Tritrichomonas Systematics of an enigmatic genus. Mol Cell Probes. 2012; 26(3):132-136. https:// doi.org/10.1016/j.mcp.2012.02.002

5. Tolbert MK, Stauffer SH, Brand MD, Gookin JL. Cysteine Protease Activity of Feline Tritrichomonas foetus Promotes AdhesionDependent Cytotoxicity to Intestinal Epithelial Cells. Appleton JA, editor. Infect Immun. 2014; 82(7):2851-2859. http://iai. asm.org/lookup/doi/10.1128/IAI.01671-14

6. Šlapeta J, Müller N, Stack CM, Walker G, Lew-Tabor A, Tachezy J, et al. Comparative analysis of Tritrichomonas foetus (Riedmüller, 1928) cat genotype, T. foetus (Riedmüller, 1928) cattle genotype and Tritrichomonas suis (Davaine, 1875) at 10 DNA loci. Int J Parasitol. 2012; 42(1314):1143-1149. https://doi.org/10.1016/j. ijpara.2012.10.004 
7. Maritz JM, Land KM, Carlton JM, Hirt RP. What is the importance of zoonotic trichomonads for human health? Trends Parasitol. 2014; 30(7):333-341. http:// dx.doi.org/10.1016/j.pt.2014.05.005

8. Suzuki J, Kobayashi S, Osuka H, Kawahata D, Oishi T, Sekiguchi K, et al. Characterization of a human isolate of Tritrichomonas foetus (cattle/swine genotype) infected by a zoonotic opportunistic infection. J Vet Med Sci. 2016; 78(4):633-640. https://doi. org/10.1292/jvms.15-0644\%0A

9. Manning K. Update on the diagnosis and management of tritrichomonas foetus infections in cats. Top Companion Anim Med. 2010; 25(3):145-148. https://doi. org/10.1053/j.tcam.2010.08.001

10. Ryan CM, Miguel $\mathrm{N}$ de, Johnson PJ. Trichomonas vaginalis : current understanding of host-parasite interactions. Essays Biochem. 2011; 51:161-175. https://doi.org/10.1042/bse0510161

11. Ghabanchi J, Zibaei M, Afkar Md, Sarbazie A. Prevalence of oral Entamoeba gingivalis and Trichomonas tenax in patients with periodontal disease and healthy population in Shiraz, southern Iran. Indian J Dent Res. 2010; 21(1):89. http://www.ijdr.in/text. asp?2010/21/1/89/62821

12. Shi Y, Jiang W, Ma Z, Qiu Y. A case report of pulmonary tritrichomonosis in a pig. BMC Vet Res. 2017; 13(1):348. https://doi. org/10.1186/s12917-017-1242-x

13. Sun Z, Stack C, Šlapeta J. Sequence differences in the diagnostic region of the cysteine protease 8 gene of Tritrichomonas foetus parasites of cats and cattle. Vet Parasitol. 2012; 186(3-4):445-449. https:// doi.org/10.1016/j.vetpar.2011.12.001

14. Mueller $\mathrm{K}$, Morin-Adeline V, Gilchrist $\mathrm{K}$, Brown G, Šlapeta J. High prevalence of Tritrichomonas foetus 'bovine genotype' in faecal samples from domestic pigs at a farm where bovine trichomonosis has not been reported for over 30 years. Vet Parasitol. 2015; 212(3-4):105-110. https://doi. org/10.1016/j.vetpar.2015.08.010
15. Doi J, Hirota J, Morita A, Fukushima $\mathrm{K}$, Kamijyo $\mathrm{H}$, Ohta $\mathrm{H}$, et al. Intestinal Tritrichomonas suis ( $=\mathrm{T}$. foetus) infection in Japanese cats. J Vet Med Sci. 2012; 74(4):413-417. https://doi.org/10.1292/ jvms.11-0171

16. Šlapeta J, Craig S, McDonell D, Emery D. Tritrichomonas foetus from domestic cats and cattle are genetically distinct. Exp Parasitol. 2010; 126(2):209-213. https:// doi.org/10.1016/j.exppara.2010.04.024

17. Morin-Adeline $V$, Lomas $R, O^{\prime}$ Meally $D$, Stack C, Conesa A, Šlapeta J. Comparative transcriptomics reveals striking similarities between the bovine and feline isolates of Tritrichomonas foetus: Consequences for in silico drug-target identification. BMC Genomics. 2014; 15(1):955. https://doi. org/10.1186/1471-2164-15-955

18. Reinmann K, Müller N, Kuhnert $P$, Campero CM, Leitsch D, Hess M, et al. Tritrichomonas foetus isolates from cats and cattle show minor genetic differences in unrelated loci ITS-2 and EF-1a. Vet Parasitol. 2012; 185(24):138-144. https://doi.org/10.1016/j. vetpar.2011.09.032

19. Duboucher C, Caby S, Dufernez F, Chabé M, Gantois N, Delgado-Viscogliosi P, et al. Molecular identification of Tritrichomonas foetus-like organisms as coinfecting agents of human Pneumocystis pneumonia. J Clin Microbiol. 2006; 44(3):1165-1168. https:// doi.org/10.1128/JCM.44.3.1165-1168.2006

20. Yao C. Opportunistic Human Infections Caused by Tritrichomonas species: A Mini-Review. Clin Microbiol Newsl. 2012; 34(16):127-131. https://doi.org/10.1016/j. clinmicnews.2012.07.004 